\title{
Mechanical properties of water hyacinth fibers - polyester composites before and after immersion in water
}

\begin{abstract}
This study reported moisture absorption of untreated and treated individual water hyacinth (WH) fibers as well as comparison the mechanical properties of WH fibers $\ddot{i}$ unsaturated polyester (UPR) matrix composites after and before immersion in water. The result shows that the individual WH fibers treated with various alkali concentration did not exhibit significantly decreases of their moisture absorption. SEM photograph in cross section of the treated WH fibers shows swollen cell wall containing more nano and micro hollows. Tensile and flexure strength of the wet composite samples are lower than that of dried ones. However, increases volume fraction of the WH fibers in UPR matrix affected slightly on enhancement mechanical properties of the composite samples.
\end{abstract}

Keyword: Water hyacinth fibers; Moisture absorption; Unsaturated polyester 